\title{
Re-evaluation of microscopy confirmed Plasmodium falciparum and Plasmodium vivax malaria by nested PCR detection in southern Ethiopia
}

Seleshi Kebede Mekonnen ${ }^{1,2,3}$, Abraham Aseffa ${ }^{2}$, Girmay Medhin ${ }^{1}$, Nega Berhe ${ }^{1}$ and Thirumalaisamy P Velavan ${ }^{3^{*}}$

\begin{abstract}
Background: With $75 \%$ of the Ethiopian population at risk of malaria, accurate diagnosis is crucial for malaria treatment in endemic areas where Plasmodium falciparum and Plasmodium vivax co-exist. The present study evaluated the performance of regular microscopy in accurate identification of Plasmodium spp. in febrile patients visiting health facilities in southern Ethiopia.
\end{abstract}

Methods: A cross-sectional study design was employed to recruit study subjects who were microscopically positive for malaria parasites and attending health facilities in southern Ethiopia between August and December 2011. Of the 1,416 febrile patients attending primary health facilities, 314 febrile patients, whose slides were positive for $P$. falciparum, $P$. vivax or mixed infections using microscopy, were re-evaluated for their infection status by PCR. Finger-prick blood samples were used for parasite genomic DNA extraction. Phylogenetic analyses were performed to reconstruct the distribution of different Plasmodium spp. across the three geographical areas.

Results: Of the 314 patients with a positive thick blood smear, seven patients (2\%) were negative for any of the Plasmodium spp. by nested PCR. Among 180 microscopically diagnosed P. falciparum cases, 111 (61.7\%) were confirmed by PCR, 44 (24.4\%) were confirmed as P. vivax, 18 (10\%) had mixed infections with P. falciparum and $P$. vivax and two (1.1\%) were mixed infections with P. falciparum and $P$. malariae and five $(2.8 \%)$ were negative for any of the Plasmodium spp. Of 131 microscopically diagnosed P. vivax cases, 110 (84\%) were confirmed as P. vivax, 14 (10.7\%) were confirmed as P. falciparum, two (1.5\%) were P. malariae, three (2.3\%) with mixed infections with $P$. falciparum and $P$. vivax and two (1.5\%) were negative for any of the Plasmodium spp. Plasmodium falciparum and $P$. vivax mixed infections were observed. Plasmodium malariae was detected as mono and mixed infections in four individuals.

Conclusion: False positivity, under-reporting of mixed infections and a significant number of species mismatch needs attention and should be improved for appropriate diagnosis. The detection of substantial number of false positive results by molecular methodologies may provide the accurate incidence of circulating Plasmodium species in the geographical region and has important repercussions in understanding malaria epidemiology and subsequent control.

Keywords: Malaria, Plasmodium, Nested PCR, Microscopy, Ethiopia

\footnotetext{
* Correspondence: Velavan@medizin.uni-tuebingen.de

${ }^{3}$ Institute of Tropical Medicine, University of Tübingen, Wilhelmstraße 27,

72074 Tübingen, Germany

Full list of author information is available at the end of the article
} 


\section{Background}

Malaria is one of the major causes of mortality and morbidity in tropical and subtropical countries with an estimated 655,000 malaria deaths in 2010, and 91\% of these cases were in sub-Saharan Africa [1]. Malaria is caused by parasites of the genus Plasmodium, with four different species specifically infecting humans (Plasmodium falciparum, Plasmodium vivax, Plasmodium ovale and Plasmodium malariae) and parasites of non-human primates, such as Plasmodium knowlesi, infecting humans occasionally. Plasmodium falciparum is the most virulent species contributing to a larger extent to malarial deaths in Africa, including Ethiopia. Plasmodium vivax and $P$. ovale form resting stages in the liver as hypnozoites and can cause a clinical relapse [2].

Ethiopia's diverse topography and climatic conditions make malaria a seasonal infection covering $75 \%$ of the prefecture of the country [3] and malaria epidemic occur mostly in high and low land arid regions of the country [4]. The majority of Ethiopian regional states (Oromia, Amhara, Tigray, South Nation, and Nationalities, and People's Region (SNNPR)) have experienced the 1998 malarial epidemic [5]. Plasmodium falciparum accounts for $60-70 \%$ and $P$. vivax accounts for $30-40 \%$ of malaria cases in Ethiopia, although these proportions might fluctuate on the spatial and temporal scale [6]. Anopheles arabiensis is the main vector widely distributed in Ethiopia, while Anopheles pharoensis, Anopheles funestus and Anopheles nili contribute as secondary vectors [7].

Hospitals and health centres in Ethiopia diagnose malaria by microscopy, whereas the health posts employ rapid diagnostic tests (RDT) for diagnosis of P. falciparum and $P$. vivax [3]. The use of RDTs in malarial diagnosis does not require skilled personnel compared to microscopy and reduces inappropriate use of anti-malarial drugs [4]. However, RDTs do not allow quantification and differentiation of different Plasmodium species other than P. falciparum and $P$. vivax. Microscopy remains the golden standard of choice to determine the disease burden. Additionally microscopy can identify febrile patients negative for any Plasmodium infection, thereby directing patients towards an alternative treatment algorithm $[5,8]$.

Microscopy is a valuable technique and has been shown to detect about $75 \%$ of malaria infections in high transmission areas and miss up to $88 \%$ of infections in low transmission areas [9]. The detection of low numbers of parasites, and the labour-intensive procedure remain a hurdle in differentiating Plasmodium species by microscopy [10]. In endemic regions, $P$. malariae and $P$. ovale infections are frequently overlooked. Dedicated laboratory personnel with good experience remain the key for accurate diagnosis of $P$. malariae and $P$. ovale in areas where $P$. falciparum and $P$. vivax predominate. Prompt and accurate diagnosis followed by an effective treatment largely depends on the skills of the laboratory personnel, who can spend appropriate time in examining blood film. On the other hand, microscopy techniques fail to detect mixed infections, when one of the Plasmodium species is present at low levels.

In recent years, molecular detection of Plasmodium with higher sensitivity is gaining importance for accurate detection. Methods such as nested PCR are capable of detecting few parasites/ $\mu$ in blood [10-15]. The nested PCR approach identifies the species-specific Plasmodium DNA by amplifying the $18 \mathrm{~s}$ ribosomal RNA region of the parasite [11]. The ribosomal RNA genes in Plasmodium are four to eight copies per haploid genome and are scattered on different chromosomes, with two distinct subgroups whose expression is regulated both by type $\mathrm{A}$ and type $\mathrm{B}$ genes that are expressed in the asexual and sexual stages in the vertebrate host, respectively [16,17]. The nucleotide sequence of the SSUrRNA is largely conserved between Plasmodium and different species reveal genetic heterogeneity in their respective ribosomal regions. This variation, together with the abundance of ribosomes in the parasite, has led to the development of effective diagnostic probes based on SSUrRNA sequences [18].

In Ethiopia, previous reports on malaria surveillance were based on microscopic results. The majority of these studies were conducted either with little or no information or with few technical resources [19-21]. In the current study, the accuracy of results obtained by routine microscopy performed by experienced laboratory personnel was compared to molecular detection methodologies. These microscopists were experienced and were supervised by fellow senior laboratory technologists. In addition, they received additional training on a regular basis to improve their detection skills on differentiating Plasmodium spp. Additionally, 18SrRNA genes were subsequently sequenced and phylogenetic relationship was investigated to determine the genetic diversity of the Plasmodium spp.

\section{Methods}

\section{Study area and design}

A health institution-based study targeting febrile patients attending primary health facilities was conducted in southern Ethiopia between August and December, 2011. Malaria transmission in Ethiopia is seasonal with higher transmission peaks in the months between September and December, and between April and May following rainy seasons. The three study sites included in this study were Omo Nada, Bala Wajo and Arba Minch.

\section{Study participants}

Study participants were recruited from the routine health delivery system. Self-presenting febrile patients attending health centres in Omo Nada $(n=713)$, Bala Wajo $(n=312)$ and Arba Minch $(n=391)$ and whose age was at least six months between August and December 2011 were eligible 
for the current study. Any patient reported as being infected either with $P$. falciparum or $P$. vivax or with a mixed infection using the existing health care delivery system within the target health centres was approached for consent immediately after receiving their laboratory result. For those who consented to be part of the study, approximately 5-10 $\mu$ l finger-prick blood sample was collected. No additional clinical assessment was carried out at the time of recruitment.

\section{Sampling}

In total, 314 finger-prick blood samples were collected for molecular diagnosis from microscopy-positive outpatients in Omo Nada $(\mathrm{n}=132)$, Bala Wajo $(\mathrm{n}=99)$ and Arba Minch $(n=83)$ from all patients attending the health facilities during the study period. Blood samples were collected in Whatman $3 \mathrm{~mm}$ filter papers. These patients were positive in the routine health delivery system using microscopy either for $P$. falciparum or $P$. vivax. On collection, the blood spots were air-dried and stored in a separate, clean, sealed, plastic pouch at room temperature for further analysis.

\section{Ethics}

Ethical clearances were obtained from the institutional review boards of Aklilu Lemma Institute of Pathobiology, Jimma University and Armauer Hansen Research Institute. Informed written consent and assent (in the case of children between 12 and 18 years of age) were obtained from each patient or a parent/legal guardian. The study participation was voluntary and had no influence on the treatment provision at the respective health facilities. All patient information were kept confidential.

\section{Blood film management and patient care}

Giemsa-stained thick and thin blood films are routinely used in Ethiopian hospitals and health centres for the detection of Plasmodium without any quantitative estimation of parasitaemia. In the current study, thin and thick blood films were prepared from finger-prick blood and air-dried, fixed in methanol and subsequently stained for 20-30 minutes in Giemsa. At least 100 thick field films with an oil immersion lens at 100X magnification were examined before a slide was considered negative. All individuals positive for $P$. falciparum were treated with artemether-lumefantrine (AL), $P$. vivax-positive patients were treated with chloroquine and mixed infections were treated with AL.

\section{DNA extraction and molecular detection}

Genomic DNA was isolated using QIAgen DNA Mini Kit blood and tissue (QIAGEN, Germany) according to the manufacturer's instructions. The extracted DNA was stored at $-20^{\circ} \mathrm{C}$ until use. Nested PCR assay were carried out as previously described elsewhere [20]. DNA samples were amplified by species-specific primer pairs designed to amplify small subunit ribosomal ribonucleic acid (ssrRNA) genes of $P$. falciparum, $P$. vivax, $P$. malariae and $P$. ovale (Table 1 ). In brief, both the primary and nested amplifications were carried out in a $20 \mu \mathrm{l}$ reaction volume containing $1 \mathrm{X}$ buffer, $2.5 \mathrm{mM} \mathrm{MgCl} 2,200 \mu \mathrm{M}$ dNTPs, $200 \mathrm{nM}$ primers, and 1U Taq DNA-polymerase with approximately $10 \mathrm{ng}$ of DNA template on a PTC-200 Thermal cycler (MJ Research, USA). The Plasmodium genus-specific amplification was followed by $P$. falciparum, $P$. vivax, $P$. malariae and $P$. ovale species-specific PCR amplification. Thermal cycling parameters for first round of amplification were: initial denaturation at $94^{\circ} \mathrm{C}$ for $5 \mathrm{~min}$, followed by 40 cycles of, respectively, $25 \mathrm{sec}$ at $94^{\circ} \mathrm{C}$ for denaturation, $25 \mathrm{sec}$ for $58^{\circ} \mathrm{C}$ for annealing temperature, and $60 \mathrm{sec}$ at $72^{\circ} \mathrm{C}$ extension and the thermal parameters for the second PCR were followed by 30 cycles of, respectively, $25 \mathrm{sec}$ at $94^{\circ} \mathrm{C}$ for denaturation, $25 \mathrm{sec}$ for $56^{\circ} \mathrm{C}$ for annealing temperature, and $60 \mathrm{sec}$ at $72^{\circ} \mathrm{C}$ extension. This was followed by a final extension of $10 \mathrm{~min}$ at $72^{\circ} \mathrm{C}$. In each run negative and positive controls were included. Genomic DNA from healthy as well as from individuals who had not travelled to malaria-endemic areas were included as negative controls in all PCR diagnostic assays. Amplicons were separated on a $1.2 \%$ agarose gel electrophoresis run along with a 100 bp DNA ladder (Invitrogen, Karlsruhe, Germany). The presence or absence of different Plasmodium species was confirmed with representative amplicon size that were species-specific. Samples that failed to amplify were subjected to repeated amplification procedures with different PCR additives.

\section{Sequencing and phylogenetic analysis}

Thirty-one $(\mathrm{n}=31)$ Plasmodium-positive samples were further selected to determine the genetic diversity of the existing subspecies from three collected sites. The selected Plasmodium samples included 11 P. falciparum (three from Omo Nada; two from Bala Wajo; six from Arba Minch), 16 P. vivax (nine from Omo Nada; four from Bala Wajo; three from Arba Minch) and four P. malariae from Omo Nada. PCR products were cleaned up using ExoSAP-IT (USB, Affymetrix, USA) and $1 \mu \mathrm{l}$ of the purified product were directly used as templates for sequencing, using the BigDye terminator v. 2.0 cycle sequencing kit (Applied Biosystems, USA) on a ABI 3130XL DNA sequencer, according to the manufacturer's instructions.

Five 18srRNA-specific DNA sequences for each Plasmodium (P. falciparum JQ627152.1, P. vivax HF945443.1, HF945441.1 and JQ627152.1 and P. malariae KC906731.1) obtained from NCBI database were aligned with 31 samples (11 P. falciparum samples, $16 P$. vivax samples and four $P$. malariae samples) sequenced for 18srRNA using Codon code Aligner 4.0 software. The phylogenetic tree 
Table 1 Species-specific primers used for nested PCR and sequencing

\begin{tabular}{|c|c|c|c|}
\hline Malaria species & Primer & Primer sequence $5^{\prime}-3^{\prime}$ & Size (bp) \\
\hline \multirow[t]{2}{*}{ Plasmodium } & rPLU5 & CCTGTTGTTGCCTTAAACTTC & 1,100 \\
\hline & rPLU6 & TTAAAATTGTTGCAGTTAAAAC & \\
\hline \multirow[t]{2}{*}{ P. falciparum } & rFAL1 & & 250 \\
\hline & rFAL2 & ACACAATGAACTCAATCATGACTACCCGTC & \\
\hline \multirow[t]{2}{*}{ P. vivax } & rVIV1 & CGCTTCTAGCTTAATCCACATAACTGATAC & 120 \\
\hline & rVIV2 & ACTTCCAAGCCGAAGCAAAGAAAGTCCTTA & \\
\hline \multirow[t]{2}{*}{ P. malariae } & rMAL1 & ATAACATAGTTGTACGTTAAGAATAACCGC & 144 \\
\hline & rMAL2 & AAAATTCCCATGCATAAAAAATTATACAAA & \\
\hline \multirow[t]{2}{*}{ P. ovale } & rOVA1 & ATCTCTITGCTATIIITAAGTATTGGAGA & 800 \\
\hline & rOVA2 & GAAAAGGACACATTAATTGTATCCTAGTG & \\
\hline
\end{tabular}

was reconstructed by Maximum Likelihood method based on Kimura 2-parameter model using the MEGA ver5.2 software [22] with 1000 bootstrap iterations.

\section{Results}

Study subject characterization

Blood films were examined from a total of 314 study participants aged at least six months for the presence of malaria parasites, using microscopy as well as by speciesspecific nested PCR method. Among the study participants, $21 \%$ were children less than four years of age, $60.2 \%$ were males and $38.9 \%$ were females. Of these study participants, 307 were reconfirmed positive for the presence of Plasmodium DNA by PCR. Of 314 study subjects, $21 \%$ were children less than four years of age, 189(60.2\%) were male and $125(38.9 \%)$ were female. Among 307 PCR-confirmed cases, $125(40.7 \%)$ were positive for $P$. falciparum, 154 (50.2\%) were positive for P.vivax, two (0.7\%) were positive for $P$. malariae, 24(7.8\%) were positive for $P$. falciparum and $P$. vivax double infections, two $(0.7 \%)$ were positive for $P$. falciparum and $P$. malariae double infection. Seven study participants remained negative for all species investigated (Table 2).

All single and mixed infections of $P$. malariae were not detected by the golden standard microscopic examination. Most of the Plasmodium-infected individuals belong to the age group between five and 14 years $(n=154$,
$49 \%)$. Of these 154 individuals aged from 5 to 14 years, 81 (52.6\%) were infected by $P$. vivax and 55 (35.7\%) infected by $P$. falciparum. A total of 66 (21\%) were children less than four years. The ages of all $P$. malariae-positive cases that were detected by PCR were below 28 years (Table 2 ).

\section{Comparison of molecular diagnosis with microscopy}

Comparison of results from microscopy and nested PCR is summarized in Table 3. Microscopic examination of blood films reported cases of $P$. falciparum (180 positive cases), cases of $P$. vivax (131 positive cases) in all the study sites. Three mixed infections of P. falciparum and $P$. vivax were detected in the samples from Omo Nada. In relative terms the highest numbers of $P$. falciparum and $P$. vivax patients were reported from Omo Nada site. Of all 180 microscopically confirmed $P$. falciparum cases, 111 cases $(62 \%)$ were mono-infections for $P$. falciparum, 44 cases (24\%) were $P$. vivax mono-infections, 18 cases $(10 \%)$ were double infections with $P$. falciparum and $P$. vivax, two cases (1\%) were double infections with P. falciparum and P. malariae, and five cases (3\%) were confirmed negative for any Plasmodium species by the nested PCR. Of all 131 microscopically confirmed $P$. vivax cases, 110 cases (84\%) were $P$. vivax mono-infections, 14 cases $(11 \%)$ were mono-infections for $P$. falciparum, two cases (2\%) were detected for P. malariae mono-infection, three cases $(2 \%)$ were double infections with $P$. falciparum

Table 2 Malaria parasite species stratified by sex and age among 314 study participants in southern Ethiopia

\begin{tabular}{|c|c|c|c|c|c|c|c|c|}
\hline & & Pf (\%) & $\operatorname{Pv}(\%)$ & Pm (\%) & $P f+P v(\%)$ & $P f+P m(\%)$ & Negative (\%) & Total (\%) \\
\hline \multirow[t]{2}{*}{ Sex } & Male & 75 (39.7) & $91(48.1)$ & $2(1.1)$ & $16(8.5)$ & $1(0.5)$ & $4(2.1)$ & $189(60.2)$ \\
\hline & Female & $50(40)$ & $63(50.4)$ & $0(0)$ & $8(6.4)$ & $1(0.8)$ & $3(2.4)$ & 125 (38.9) \\
\hline \multirow[t]{5}{*}{ Age group (years) } & $\leq 4$ & $29(43.9)$ & $32(48.5)$ & $1(1.5)$ & $4(6.1)$ & $0(0)$ & $0(0.0)$ & $66(21.0)$ \\
\hline & $5-14$ & $55(35.7)$ & $81(52.6)$ & $1(0.6)$ & $12(7.8)$ & $1(0.6)$ & $4(2.6)$ & $154(49.0)$ \\
\hline & $15-28$ & $22(37.3)$ & $28(47.5)$ & $0(0)$ & $5(8.5)$ & $1(1.7)$ & $3(5.1)$ & $59(17.8)$ \\
\hline & $29-45$ & $13(46.4)$ & $12(42.9)$ & $0(0)$ & $3(10.7)$ & $0(0)$ & $0(0.0)$ & $28(8.9)$ \\
\hline & $>46$ & $6(85.7)$ & $1(14.3)$ & $0(0)$ & $0(0)$ & $0(0)$ & $0(0.0)$ & $7(2.2)$ \\
\hline
\end{tabular}

$P f=P$. falciparum, $P v=P$. vivax,$P m=P$. malariae, $P o=P$. ovale,$P f+P v=P$. falciparum and $P$. vivax,$P f+P m=P$. falciparum and $P$. malariae. 
Table 3 Comparison of microscopy and nested PCR in the diagnosis of malaria in southern Ethiopia

\begin{tabular}{|c|c|c|c|c|c|c|c|}
\hline \multirow[t]{2}{*}{ Microscopy } & \multicolumn{7}{|c|}{ Nested PCR } \\
\hline & P. falciparum & P. vivax & P. malariae & $P$. falciparum $+P$. vivax & P. falciparum $+P$. malariae & Negative & Total \\
\hline P. falciparum & 111 & 44 & 0 & 18 & 2 & 5 & 180 \\
\hline P. vivax & 14 & 110 & 2 & 3 & 0 & 2 & 131 \\
\hline P. falciparum + P. vivax & 0 & 0 & 0 & 3 & 0 & 0 & 3 \\
\hline Total & 125 & 154 & 2 & 24 & 2 & 7 & 314 \\
\hline
\end{tabular}

and $P$. vivax and two cases $(2 \%)$ were confirmed negative for any Plasmodium species by the nested PCR. Molecular detection by nested PCR identified that many of the $P$. vivax cases $(44 / 180)$ were incorrectly diagnosed as $P$. falciparum by microscopy. A total of seven negative cases are observed. These seven negative cases were reconfirmed for their status by amplification for Pfmdr1 and Pfcrt alleles. P. ovale was not detected either by microscopy or by nested PCR. In total, 23 mixed infections were detected by nested PCR compared to microscopy-based detection.

\section{Gametocyte identification using microscopy}

Of all the 314 study subjects, 32 (10.2\%) carried gametocytes. Of which, 17(5.5\%) were $P$. vivax and 15 (4.8\%) were $P$. falciparum gametocyte stage. All the gametocyte stages were identified from 307 study subjects positive for Plasmodium parasites.

\section{Phylogenetic analysis}

The result of 31 Ethiopian specific isolates (11 P. falciparum isolates, $16 P$. vivax isolates and four $P$. malariae isolates) originated from different geographical areas and sequenced for 18srRNA, is summarized in Table 4. The identified nucelotide substitutions including the insertions and deletions observed in different Plasmodium isolates is summarized in Table 5 . The reconstructed phylogenetic tree inferred two distinct clades. All $P$. vivax isolates from Ethiopia matched in one group whereas all the P. falciparum and $P$. malariae clustered in another clade. Within the main clade, $P$. vivax clustered into three, $P$. falciparum clustered into two and $P$. malariae placed into one subcluster (Figure 1). The first subcluster with nine isolates contained 70 single nucleotide polymorphisms (SNP), the

Table 4 Parasite isolates utilized for phylogenetic analysis from the three study sites

\begin{tabular}{lllll}
\hline & \multicolumn{4}{c}{ Geographical regions } \\
\cline { 2 - 5 } & $\begin{array}{l}\text { Omo Nada } \\
\text { (ID) }\end{array}$ & $\begin{array}{l}\text { Bala Wajo } \\
\text { (ID) }\end{array}$ & $\begin{array}{l}\text { Arba Minch } \\
\text { (ID) }\end{array}$ & Total \\
\hline P. falciparum & $3(\mathrm{~N} 21-\mathrm{N} 23)$ & $2(\mathrm{~B} 3-\mathrm{B} 4)$ & $6(\mathrm{~A} 3-\mathrm{A} 6, \mathrm{~A} 8-\mathrm{A} 9)$ & 11 \\
P. vivax & $9(\mathrm{~N} 25-\mathrm{N} 33)$ & $4(\mathrm{~B} 6-\mathrm{B} 9)$ & $3(\mathrm{~A} 10-\mathrm{A} 11, \mathrm{~A} 17)$ & 16 \\
P. malariae & $4(\mathrm{~N} 41-\mathrm{N} 44)$ & 0 & 0 & 4 \\
\hline
\end{tabular}

second subcluster included 150 SNPs and the third subcluster contained 53 SNPs.

\section{Discussion}

Early diagnosis and prompt treatment is crucial in controlling malaria infections. Microscopic diagnosis and RDTs are widely used methods for malaria surveillance in Ethiopia. The most fatal species, P. falciparum, must be rightly differentiated from the other Plasmodium species (especially $P$. vivax), thereby directing febrile patients towards prompt treatment that can save lives. The rationale of the study was to assess species mismatch and the specificity of microscopy in differentiating Plasmodium spp. in regions where two virulent species of Plasmodium, namely $P$. falciparum and $P$. vivax, co-exist.

In Ethiopia, P. falciparum and $P$. vivax are the predominant parasite with a prevalence of 55 and $45 \%$, respectively [23]. In most endemic regions across Ethiopia both Plasmodium co-exist. The present study report a prevalence of $P$. vivax (55\%) remained high compared to $P$. falciparum (45\%) during this season. The rationale of the study was to evaluate the limitations of microscopy in differentiating Plasmodium in Ethiopia that may contribute to improve individual's life by an appropriate administration of anti-malarials. When slide positive cases

Table 5 Ethiopian Plasmodium species with their single nucleotide polymorphism

\begin{tabular}{|c|c|c|c|c|}
\hline & Plasmodium no & Insertion & Deletion & Substitution \\
\hline \multirow{3}{*}{$\mathrm{Pf}$} & N21 & 1 & 3 & 18 \\
\hline & A9 & 1 & 3 & 14 \\
\hline & N23 & 1 & 3 & 14 \\
\hline \multirow{3}{*}{ Pv sub cluster 1} & $\mathrm{~B} 6$ & 6 & 0 & 26 \\
\hline & B8 & 5 & 0 & 24 \\
\hline & B7 & 5 & 0 & 23 \\
\hline \multirow{3}{*}{ Pv sub cluster 2} & N32 & 0 & 7 & 4 \\
\hline & N29 & 0 & 7 & 4 \\
\hline & A11 & 0 & 7 & 4 \\
\hline Pv sub cluster 3 & B9 & 2 & 0 & 1 \\
\hline
\end{tabular}

$P f=P$. falciparum, $P v=P$. vivax, $P m=P$. malariae, $P o=P$. ovale, $P f+P v=P$. falciparum and $P$. vivax, $P f+P m=P$. falciparum and $P$. malariae. 


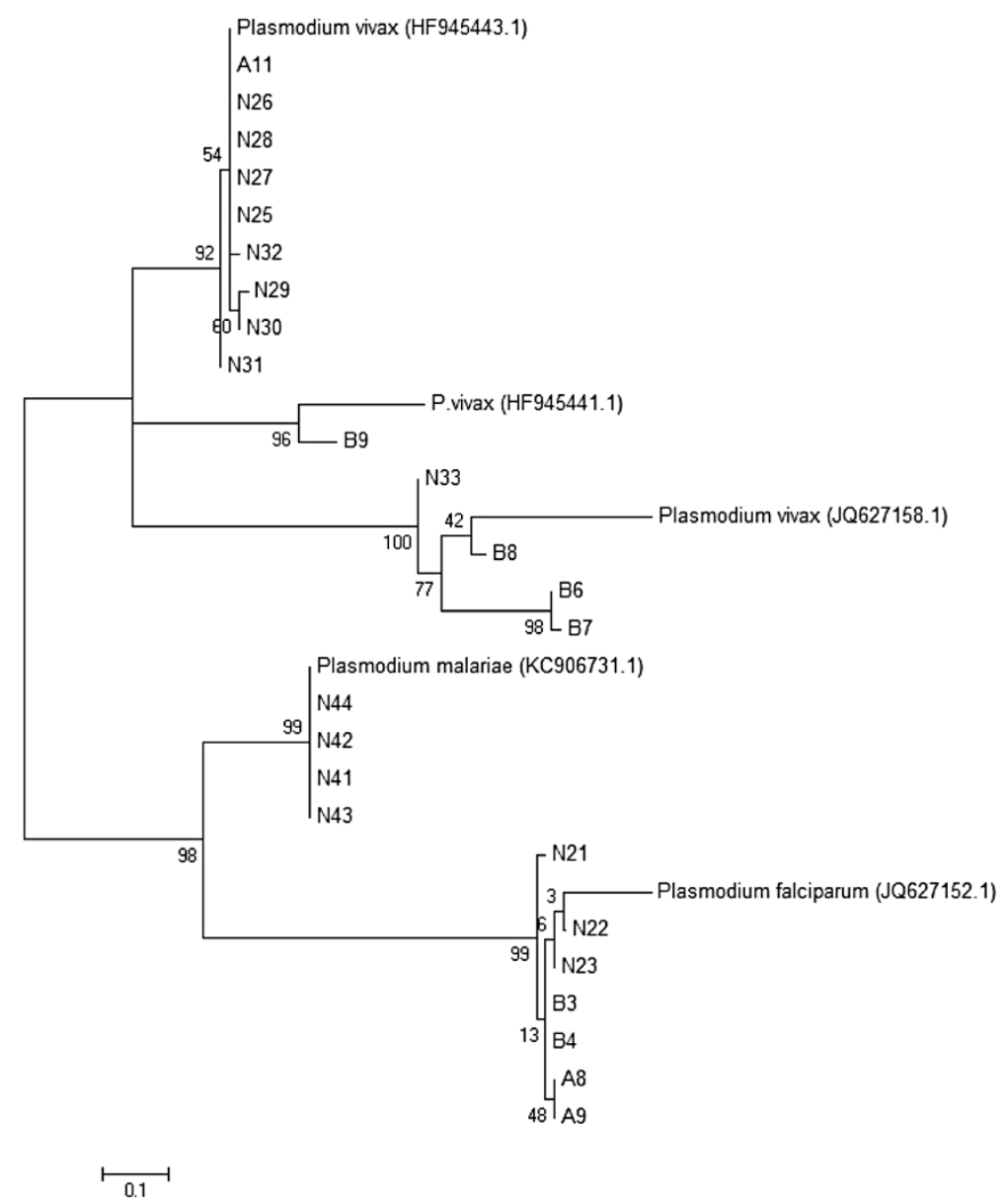

Figure 1 Reconstructed phylogenetic tree using $18 \mathrm{~S}$ ribosomal RNA nucleotide sequence from Plasmodium field isolates.

were compared by molecular detection methodology, a high proportion of $P$. vivax individuals were misdiagnosed as $P$. falciparum-positive and were treated with AL, a drug of choice in treating $P$. falciparum infections. However the treatment of 14 cases (11\%) microscopically diagnosed as $P$. vivax were mono-infections for $P$. falciparum. These results imply that these individuals were mistreated with chloroquine rather with AL. This may have had a considerable impact on treatment failure as they were misdiagnosed microscopically. Such a scenario may lead to severe malaria-related ailments and to the extent of mortality in these 14 cases. All the laboratories in the study area did not use buffer when preparing Giemsa staining solution. This might be one of the reasons for a high report of species mismatch and low sensitivity of microscopy. $\mathrm{pH}$ is an important component of Giemsa stain and $\mathrm{pH} 7.2$ is recommended, as it highlights the Schüffner's dots of $P$. vivax and Maurer's clefts of $P$. falciparum, which are essential characteristics for malaria diagnosis by microscopy.

Additionally, microscopy methodologies failed to detect multiple infections. As demonstrated by a plethora of other studies that compared microscopy with molecular diagnosis [12,24-30], nucleic acid testing by the nested PCR approach provides an accurate differentiation of Plasmodium, and that helps to rule out false positives and mixed infections [10,31]. A high prevalence of mixed infections, especially with $P$. falciparum and $P$. vivax, irrespective of study sites were observed. Of the 21 individuals with double infections, three were reported as $P$. vivax and 18 were $P$. falciparum-positive by microscopy. The reason why microscopy failed to detect mixed infections could be due to the presence of higher numbers of parasites of one species relative to the other. Moreover, in laboratories with overwhelming numbers of patients, the workload is not proportional to the laboratory staff involved. Therefore, this leads the laboratory personnel not to utilize adequate time to examine the blood film.

Although $P$. ovale is one of the prevalent parasites in western Africa [32], no individuals infected with $P$. ovale were observed in any of the studied three sites. Additionally, $P$. malariae, a species which is less common in Ethiopia, was detected in four individuals with two as mono-infection and two as double infection with $P$. falciparum. A total of seven (2\%) negative cases were 
observed. Of these individuals, five were treated for $P$. falciparum and two for $P$. vivax. The false positive cases were reconfirmed as negative for other Plasmodium spp. additionally; these false positive samples were subjected to subsequent amplification for both $p f m d r 1$ and pfcrt alleles. Microscopy failed to detect $P$. malariae mono-infections and mixed infections. The dominance of either $P$. falciparum or $P$. vivax can possibly be a reason for the failure to detect $P$. malariae by microscopy. In addition, the pattern of fever and duration of the erythrocyte 72-hour developmental cycle may contribute to $P$. malariae low numbers. Although microscopy remains as a gold standard, it has its limitations to differentiate Plasmodium spp., in addition to low parasite counts [33]. Factors influencing the precision of bloodsmear diagnosis including the experience and motivation of technicians, smear quality, the inability to determine parasite species at low parasitemia, the loss of slide quality with time and the quality of the reagents. Artifacts resembling malaria are common. Currently RDTs are widely used in malaria surveillance programmes to differentiate only $P$. falciparum or $P$. vivax infections [34].

Many studies within Ethiopia reported conflicting results on the presence of $P$. malariae prevalence, including Federal Ministry of Health of Ethiopia. A few of these reported the presence only of $P$. falciparum and $P$. vivax [6], whereas other reports demonstrated the prevalence of P. malariae in addition to the two dominant species in the country [35]. A study from Thailand reported that fever induced by low parasitaemia by $P$. vivax might limit parasitaemia and the pathogenic potential of $P$. falciparum [35]. In addition, the role of P. malariae in the aetiology of human glomerulonephritis is based on circumstantial clinical and epidemiological evidence and on renal biopsies showing granular immune deposits in the glomeruli [36].

Microscopic diagnosis of malaria is the most widely used approach for epidemiological studies. The blood smear provides much vital information that helps to correlate the parasite density to clinical malaria. Unfortunately, microscopy detection has both qualitative and quantitative limitations. Blood-smear microscopy reaches its limit of detection when parasitaemia falls below 40 infected red blood cells (IRBC) per $\mu$ l of blood $\left(10^{8}\right.$ total body parasites), and the reproducibility of parasite counts and species identification is frequently inconsistent [37]. Factors influencing the precision of microscopy-based diagnosis largely depend on high experience, motivation of technicians, due diligence in examination over an adequate period of time, smear and slide quality, and differentiation from artifacts resembling malaria [38]. False positives are common in laboratories with high patient flow and when the numbers of laboratory personnel are not proportional to the laboratory workload. Difficulties are compounded when infections contain more than one parasite species. The other disadvantage of a false positive microscopic result is patients being exposed to drugs leading to unnecessary side effects and drug wastage. Patients will also be delayed from early treatment for other infections and that can be life threatening [39].

The phylogenetic relatedness of $P$. vivax and $P$. falciparum isolates is rather not restricted to one region, but seems to be widespread across different geographical regions within Ethiopia as the isolates from Abra Minch are also found in homology to isolates from Omo Nada. All isolates of $P$. malariae were represented from the site Omo Nada and were clustered together. Moreover, $P$. vivax showed a more diverse population than $P$. falciparum and P. malariae.

\section{Conclusions}

False positivity, under-reporting of mixed infections and a significant number of species mismatch needs attention and should be improved for accurate diagnosis. Efforts must continue to re-train laboratory staff and provision of appropriate reagents based on the standard staining procedure for the diagnosis of Plasmodium parasite shall improve diagnosis. Overall, the finding of the current study highlights an important concern in the diagnosis of malaria by microscopy alone in southern Ethiopia and has important repercussions in understanding malaria epidemiology and subsequent control.

\section{Competing interests}

The authors have declared that they have no competing interests.

\section{Authors' contributions}

SK designed and performed the field study and experiments with drafting first draft. NB, AA and GM contributed to the study design and study samples and revisions of MS. TPV designed the experiments, contributed to materials and tools, supervised the experiments, data analysis, writing of the MS. All authors read and approved the final manuscript.

\section{Acknowledgements}

We thank all our study volunteers. We are grateful to all the health personnel at the sites for their extensive help in the study. A special thanks to Abebaw Tiruneh, Jihad Kemal, Albert Lalremruata, Bereket Workalemaw and Nasir Abdo. We thank Justin Antony, Felix and Fanny for sharing their expertise. SK was supported by a DAAD fellowship. The study was supported by internal grant to author TPV and sampling procedures were supported by funds from Addis Ababa University and the Sida and NORAD core grant to the Armauer Hansen Research Institute. The authors extend their thanks to Benjamin Mordmüller for his valuable comments on this manuscript. The authors acknowledge the support by the Deutsche Forschungsgemeinschaft (DFG) and Open Access Publishing Fund of Tuebingen University.

\section{Author details}

${ }^{1}$ Aklilu Lemma Institute of Pathobiology, Addis Abba University, Addis Abba, Ethiopia. ${ }^{2}$ Armauer Hansen Research Institute, Addis Abba, Ethiopia. ${ }^{3}$ Institute of Tropical Medicine, University of Tübingen, Wilhelmstraße 27, 72074 Tübingen, Germany.

Received: 12 November 2013 Accepted: 4 February 2014

Published: 6 February 2014 


\section{References}

1. Dye C, Mertens T, Hirnschall G, Mpanju-Shumbusho W, Newman RD, Raviglione MC, Savioli L, Nakatani H: WHO and the future of disease control programmes. Lancet 2013, 381:413-418.

2. Roughton SA, Green AD: Plasmodium knowlesi malaria: assessing the risk to the British Armed Forces. J R Army Med Corps 2012, 158:318-321.

3. Endeshaw T, Gebre T, Ngondi J, Graves PM, Shargie EB, Ejigsemahu Y, Ayele B, Yohannes G, Teferi T, Messele A, Zerihun M, Genet A, Mosher AW, Emerson PM, Richards FO: Evaluation of light microscopy and rapid diagnostic test for the detection of malaria under operational field conditions: a household survey in Ethiopia. Malar J 2008, 7:118.

4. Ashton RA, Kefyalew T, Tesfaye G, Counihan H, Yadeta D, Cundill B, Reithinger R, Kolaczinski JH: Performance of three multi-species rapid diagnostic tests for diagnosis of Plasmodium falciparum and Plasmodium vivax malaria in Oromia Regional State Ethiopia. Malar J 2010, 9:297.

5. Moges B, Amare B, Belyhun Y, Tekeste Z, Gizachew M, Workineh M, Gebrehiwot A, Woldeyohannes D, Mulu A, Kassu A: Comparison of CareStart HRP2/pLDH COMBO rapid malaria test with light microscopy in north-west Ethiopia. Malar J 2012, 11:234.

6. Woyessa A, Deressa W, Ali A, Lindtjorn B: Prevalence of malaria infection in Butajira area, south-central Ethiopia. Malar J 2012, 11:84.

7. Hamusse SD, Balcha TT, Belachew T: The impact of indoor residual spraying on malaria incidence in East Shoa Zone Ethiopia. Glob Health Action 2012, 5:11619.

8. Xiaodong S, Tambo E, Chun W, Zhibin C, Yan D, Jian W, Jiazhi W, Xiaonong Z: Diagnostic performance of CareStart malaria HRP2/pLDH (Pf/pan) combo test versus standard microscopy on falciparum and vivax malaria between China-Myanmar endemic borders. Malar J 2013, 12:6.

9. Okell LC, Ghani AC, Lyons E, Drakeley CJ: Submicroscopic infection in Plasmodium falciparum-endemic populations: a systematic review and meta-analysis. J Infect Dis 2009, 200:1509-1517.

10. Fontecha GA, Mendoza M, Banegas E, Poorak M, De Oliveira AM, Mancero T, Udhayakumar V, Lucchi NW, Mejia RE: Comparison of molecular tests for the diagnosis of malaria in Honduras. Malar J 2012, 11:119.

11. Snounou G, Viriyakosol S, Jarra W, Thaithong S, Brown KN: Identification of the four human malaria parasite species in field samples by the polymerase chain reaction and detection of a high prevalence of mixed infections. Mol Biochem Parasitol 1993, 58:283-292.

12. Coleman RE, Sattabongkot J, Promstaporm S, Maneechai N, Tippayachai B, Kengluecha A, Rachapaew N, Zollner G, Miller RS, Vaughan JA, Thimasarn K, Khuntirat B: Comparison of PCR and microscopy for the detection of asymptomatic malaria in a Plasmodium falciparum/vivax endemic area in Thailand. Malar J 2006, 5:121

13. Fuehrer HP, Fally MA, Habler VE, Starzengruber P, Swoboda P, Noedl H: Novel nested direct PCR technique for malaria diagnosis using filter paper samples. J Clin Microbiol 2011, 49:1628-1630.

14. Rantala AM, Taylor SM, Trottman PA, Luntamo M, Mbewe B, Maleta K, Kulmala T, Ashorn P. Meshnick SR: Comparison of real-time PCR and microscopy for malaria parasite detection in Malawian pregnant women. Malar J 2010, 9:269.

15. Taylor BJ, Martin KA, Arango E, Agudelo OM, Maestre A, Yanow SK: Real-time PCR detection of Plasmodium directly from whole blood and filter paper samples. Malar J 2011, 10:244

16. Ayele DG, Zewotir TT, Mwambi HG: Prevalence and risk factors of malaria in Ethiopia. Malar J 2012, 11:195.

17. Corredor V, Enea V: The small ribosomal subunit RNA isoforms in Plasmodium cynomolgi. Genetics 1994, 136:857-865.

18. McCutchan TF, de la Cruz VF, Lal AA, Gunderson JH, Elwood HJ, Sogin ML Primary sequences of two small subunit ribosomal RNA genes from Plasmodium falciparum. Mol Biochem Parasitol 1988, 28:63-68.

19. Abay SM, Tilahun M, Fikrie N, Habtewold A: Plasmodium falciparum and Schistosoma mansoni coinfection and the side benefit of artemetherlumefantrine in malaria patients. J Infect Dev Ctries 2013, 7:468-474.

20. Ketema T, Bacha K: Plasmodium vivax associated severe malaria complications among children in some malaria endemic areas of Ethiopia. BMC Public Health 2013, 13:637.

21. Sleshi M, Animut A, Mohammed H, Medhin G, Kebede A: Malaria microscopy performance in self-presenting febrile patients at four health facilities in Fentale district of East Shewa, Ethiopia. Ethiop Med J 2012, 50:315-324
22. Tamura K, Peterson D, Peterson N, Stecher G, Nei M, Kumar S: MEGA5: molecular evolutionary genetics analysis using maximum likelihood, evolutionary distance, and maximum parsimony methods. Mol Biol Evol 2011, 28:2731-2739.

23. Woyessa A, Hadis M, Kebede A: Human resource capacity to effectively implement malaria elimination: a policy brief for Ethiopia. Int I Technol Assess Health Care 2013, 29:212-217.

24. Jamjoom MB, Azhar EA, Tonkol AK, Al-Harthi SA, Ashankyty IM: Detection of malaria in Saudi Arabia by real-time PCR. J Egypt Soc Parasitol 2006, 36:737-748

25. Lorenzetti A, Fornazari PA, Bonini-Domingos AC, de Souza Rodrigues PR, Fugikaha E, Bonini-Domingos CR, Fraga VD, Conceição LM, Rossit AR, Cavasini CE, Couto VS, Machado RL: Mixed Plasmodium falciparum infections and its clinical implications in four areas of the Brazilian Amazon region. Acta Trop 2008, 107:8-12.

26. Mens PF, Schoone GJ, Kager PA, Schallig HD: Detection and identification of human Plasmodium species with real-time quantitative nucleic acid sequence-based amplification. Malar J 2006, 5:80.

27. Mula P, Fernandez-Martinez A, De LA, Ramos JM, Reyes F, Gonzalez V, Benito A, Berzosa P: Detection of high levels of mutations involved in anti-malaria drug resistance in Plasmodium falciparum and Plasmodium vivax at a rural hospital in southern Ethiopia. Malar J 2011, 10:214.

28. Osman MM, Nour BY, Sedig MF, De BL, Babikir AM, Mohamedani AA, Mens PF: Informed decision-making before changing to RDT: a comparison of microscopy, rapid diagnostic test and molecular techniques for the diagnosis and identification of malaria parasites in Kassala, eastern Sudan. Trop Med Int Health 2010, 15:1442-1448.

29. Parajuli K, Hanchana S, Inwong M, Pukrittayakayamee S, Ghimire P. Comparative evaluation of microscopy and polymerase chain reaction (PCR) for the diagnosis in suspected malaria patients of Nepal. Nepal Med Coll J 2009, 11:23-27.

30. Tao ZY, Zhou HY, Xia H, Xu S, Zhu HW, Culleton RL, Han ET, Lu F, Fang Q, Gu YP, Liu YB, Zhu GD, Wang WM, Li JL, Cao J, Gao Q: Adaptation of a visualized loop-mediated isothermal amplification technique for field detection of Plasmodium vivax infection. Parasit Vectors 2011, 4:115.

31. Tesfaye S, Belyhun Y, Teklu T, Mengesha T, Petros B: Malaria prevalence pattern observed in the highland fringe of Butajira. Southern Ethiopia: a longitudinal study from parasitological and entomological survey. Malar J 2011, 10:153

32. May J, Mockenhaupt FP, Ademowo OG, Falusi AG, Olumese PE, Bienzle U, Meyer CG: High rate of mixed and subpatent malarial infections in southwest Nigeria. Am J Trop Med Hyg 1999, 61:339-343.

33. Mayxay M, Pukrittayakamee S, Newton PN, White NJ: Mixed-species malaria infections in humans. Trends Parasitol 2004, 20:233-240.

34. Zhou M, Liu Q, Wongsrichanalai C, Suwonkerd W, Panart K, Prajakwong S, Pensiri A, Kimura M, Matsuoka H, Ferreira MU, Isomura S, Kawamoto F: High prevalence of Plasmodium malariae and Plasmodium ovale in malaria patients along the Thai-Myanmar border, as revealed by acridine orange staining and PCR-based diagnoses. Trop Med Int Health 1998, 3:304-312.

35. Yewhalaw D, Kassahun W, Woldemichael K, Tushune K, Sudaker M, Kaba D, Duchateau L, Van Bortel W, Speybroeck N: The influence of the Gilgel-Gibe hydroelectric dam in Ethiopia on caregivers' knowledge, perceptions and health-seeking behaviour towards childhood malaria. Malar J 2010, 9:47.

36. Ehrich $\mathrm{JH}$, Eke FU: Malaria-induced renal damage: facts and myths. Pediatr Nephrol 2007, 22:626-637.

37. Ohrt C, Purnomo Sutamihardja MA, Tang D, Kain KC: Impact of microscopy error on estimates of protective efficacy in malaria-prevention trials. $J$ Infect Dis 2002, 186:540-546.

38. Golassa L, Enweji N, Erko B, Aseffa A, Swedberg G: Detection of a substantial number of sub-microscopic Plasmodium falciparum infections by polymerase chain reaction: a potential threat to malaria control and diagnosis in Ethiopia. Malar J. 2013, 12:352.

39. Andrews $L$, Andersen RF, Webster D, Dunachie S, Walther RM, Bejon P, Hunt-Cooke A, Bergson G, Sanderson F, Hill AV, Gilbert SC: Quantitative real-time polymerase chain reaction for malaria diagnosis and its use in malaria vaccine clinical trials. Am J Trop Med Hyg 2005, 73:191-198.

doi:10.1186/1475-2875-13-48

Cite this article as: Mekonnen et al:: Re-evaluation of microscopy confirmed Plasmodium falciparum and Plasmodium vivax malaria by nested PCR detection in southern Ethiopia. Malaria Journal 2014 13:48. 